\title{
Stereoselective Formation of Tri- and Tetracycles by Samarium Diiodide-Induced Cyclizations of Naphthyl Substituted Ketones
}

Mathias Berndt, Iva Hlobilová and Hans-Ulrich Reissig*

Institut für Chemie - Organische Chemie, Freie Universität Berlin, Takustr. 3, D-14195 Berlin, Germany

hans.reissig@chemie.fu-berlin.de

Experimental Conditions: All reactions were performed under argon atmosphere in flame dried flasks. Unless otherwise stated, materials were obtained from commercial suppliers and were used without further purification. Hexamethylphosphoramide was distilled and kept under argon. Tetrahydrofuran was freshly distilled from sodium/benzophenone under argon for each of the $\mathrm{SmI}_{2}$ reactions.

General experimental procedure: Samarium (2.4 - 2.5 equiv.) and 1,2-diiodoethane (2.2 equiv.) were suspended in freshly distilled anhydrous THF (10 mL per mmol samarium) under an argon atmosphere and stirred for $2 \mathrm{~h}$ at room temperature. To the resulting dark blue solution HMPA (18 equiv.) was added. The ketone and tert-butanol (2.0 equiv.), dissolved in THF $(20 \mathrm{~mL})$, were then added in one portion to the deep purple solution. After $16 \mathrm{~h}$ the reaction mixture was quenched with saturated aqueous solution of sodium bicarbonate, the organic layer was separated and the aqueous layer was extracted with diethyl ether $(3 \times 25$ $\mathrm{mL})$. The combined ether extracts were washed with brine $(25 \mathrm{~mL})$, dried over anhydrous magnesium sulfate, filtered and evaporated. The resulting crude product was purified by flash chromatography on silica gel using hexane/ethyl acetate mixtures.

\section{rac-(1S,4aS,10aS)-1-Methyl-1,2,3,4,4a,10a-hexahydrophenanthren-1-ol (8)}

Analytical data: colorless oil; ${ }^{1} \mathrm{H}$ NMR $\left(\mathrm{CDCl}_{3}, 500 \mathrm{MHz}\right): \delta=7.16-7.09$ (m, $\left.3 \mathrm{H}, \mathrm{Ar}\right)$, 7.06-7.01 (m, $1 \mathrm{H}, \mathrm{Ar}), 6.46$ (dd, $J=3.2,9.7 \mathrm{~Hz}, 1 \mathrm{H}, 9-\mathrm{H}), 5.68$ (br. d, $J=9.7 \mathrm{~Hz}, 1 \mathrm{H}, 10-$ $\mathrm{H}), 3.11(\mathrm{td}, J=5.4,12.1 \mathrm{~Hz}, 1 \mathrm{H}, 4 \mathrm{a}-\mathrm{H}), 2.57\left(\mathrm{~m}_{\mathrm{c}}, 1 \mathrm{H}, 10 \mathrm{a}-\mathrm{H}\right), 1.75-1.44\left(\mathrm{~m}, 6 \mathrm{H}, \mathrm{CH}_{2}\right.$, $\mathrm{OH}), 1.38-1.31\left(\mathrm{~m}, 1 \mathrm{H}, \mathrm{CH}_{2}\right), 1.34\left(\mathrm{~s}, 3 \mathrm{H}, \mathrm{CH}_{3}\right) ;{ }^{13} \mathrm{C} \mathrm{NMR}\left(\mathrm{CDCl}_{3}, 126 \mathrm{MHz}\right): \delta=140.6$, 132.7 ( 2 s, C-4b, C-8a), 128.8, 128.7, 128.3, 128.2, 127.6, 126.3 (6 d, C-5, C-6, C-7, C-8, C9, C-10), 71.5 (s, C-1), 46.4 (d, C-10a), 36.7 (d, C-4a), 35.0, 27.3, 20.0 (3 t, $\mathrm{CH}_{2}$ ), 28.7 (q, $\mathrm{CH}_{3}$ ); IR (film): $v=3420 \mathrm{~cm}^{-1}$ (br. s, OH), 3100-2900 (=C-H, C-H), $1600(\mathrm{C}=\mathrm{C}) ; \mathrm{C}_{15} \mathrm{H}_{18} \mathrm{O}$ (214.3) calcd.: C 84.07, H 8.47; found: C 84.22, H 84.22. 
rac-(1S,9aS)-1-Methyl-1,2,3,4,9,9a-hexahydroanthracen-1-ol (10a)

Analytical data: colorless oil; ${ }^{1} \mathrm{H}$ NMR $\left(\mathrm{CDCl}_{3}, 500 \mathrm{MHz}\right): \delta=7.39(\mathrm{~d}, J=7.3 \mathrm{~Hz}, 1 \mathrm{H}, \mathrm{Ar})$, 7.20-7.12 (m, 3 H, Ar), 5.63 (s, 1 H, 10-H), 3.44-3.24 (m, 3 H, 9-H, 9a-H), 2.33-2.28, 2.071.98 (2 m, $1 \mathrm{H}$ each, 4-H), 1.93-1.74 (m, 4 H, 2-H, 3- $\left.\mathrm{H}_{\mathrm{A}}, \mathrm{OH}\right), 1.52-1.41\left(\mathrm{~m}, 1 \mathrm{H}, 3-\mathrm{H}_{\mathrm{B}}\right), 0.84$ (s, $\left.3 \mathrm{H}, \mathrm{CH}_{3}\right) ;{ }^{13} \mathrm{C} \mathrm{NMR}\left(\mathrm{CDCl}_{3}, 126 \mathrm{MHz}\right): \delta=137.8,135.0,133.7$ (3 s, C-4a, C-8a, C-10a), 129.4, 128.3, 126.2, 125.5 (4 d, C-5, C-6, C-7, C-8), 117.9 (d, C-10), 76.5 (s, C-1), 53.0 (d, C9a), 41.0 (t, C-2), 35.4 (t, C-4), 30.3 (t, C-9), 25.6 (t, C-3), 20.9 (q, $\mathrm{CH}_{3}$ ); IR (film): v = 3450 $\mathrm{cm}^{-1}$ (br. s, OH), 3100-2850 (=C-H, C-H), $1600(\mathrm{C}=\mathrm{C}) ; \mathrm{C}_{15} \mathrm{H}_{18} \mathrm{O}$ (214.3) calcd.: C 84.07, H 8.47; found: C 84.34, H 8.47.

\section{rac-(1R,9aS)-1-Methyl-1,2,3,4,9,9a-hexahydroanthracen-1-ol (10b)}

Analytical data: colorless oil; ${ }^{1} \mathrm{H}$ NMR $\left(\mathrm{CDCl}_{3}, 500 \mathrm{MHz}\right): \delta=7.09-7.04$ (m, $\left.3 \mathrm{H}, \mathrm{Ar}\right), 6.91$ (d, $J=6.5 \mathrm{~Hz}, 1 \mathrm{H}, \mathrm{Ar}), 6.17$ (s, $1 \mathrm{H}, 10-\mathrm{H}), 3.19$ (dd, $\left.J=3.5,17.3 \mathrm{~Hz}, 1 \mathrm{H}, 9-\mathrm{H}_{\mathrm{A}}\right), 3.08$ (dd, $J$ $\left.=10.4,17.3 \mathrm{~Hz}, 1 \mathrm{H}, 9-\mathrm{H}_{\mathrm{B}}\right), 2.47(\mathrm{dd}, J=3.5,10.4 \mathrm{~Hz}, 1 \mathrm{H}, 9 \mathrm{a}-\mathrm{H}), 2.39-2.34\left(\mathrm{~m}, 1 \mathrm{H}, 4-\mathrm{H}_{\mathrm{A}}\right)$, $2.15\left(\mathrm{dt}, J=5.0,13.2 \mathrm{~Hz}, 1 \mathrm{H}, 4-\mathrm{H}_{\mathrm{B}}\right), 1.86-1.75\left(\mathrm{~m}, 3 \mathrm{H}, 3-\mathrm{H}_{\mathrm{A}}, 2-\mathrm{H}_{\mathrm{A}}, \mathrm{OH}\right), 1.61(\mathrm{dt}, J=5.2$, $\left.13.2 \mathrm{~Hz}, 1 \mathrm{H}, 2-\mathrm{H}_{\mathrm{B}}\right), 1.45$ (tq, $\left.J=4.4,13.2 \mathrm{~Hz}, 1 \mathrm{H}, 3-\mathrm{H}_{\mathrm{B}}\right), 1.01\left(\mathrm{~s}, 3 \mathrm{H}, 1-\mathrm{CH}_{3}\right) ;{ }^{13} \mathrm{C} \mathrm{NMR}$ $\left(\mathrm{CDCl}_{3}, 126 \mathrm{MHz}\right): \delta=140.8,133.4,133.4$ (3 s, C-4a, C-8a, C-10a), 127.2, 126.5, 126.0, 125.5 (4 d, C-5, C-6, C-7, C-8), 75.2 (s, C-1), 48.3 (d, C-9a), 42.8 (t, C-2), 35.0 (t, C-4), 26.2 (t, C-9), 25.0 (t, C-3), 20.6 (q, $\mathrm{CH}_{3}$ ); IR (film): $v=3450 \mathrm{~cm}^{-1}$ (br. s, OH), 3100-2850 (=C-H, C-H), $1600(\mathrm{C}=\mathrm{C}) ; \mathrm{C}_{15} \mathrm{H}_{18} \mathrm{O}$ (214.3) calcd.: C 84.07, H 8.47; found: C 84.08, H 8.25.

\section{rac-(1S,2S,11S,13S)-1-Isopropyl-15-oxatetracyclo[11.2.1.0 $\left.{ }^{2,11} .0^{5,10}\right]$ hexadeca-3,5(10),6,8-} tetraen-14-one (15)

Analytical data: colorless solid; $\mathrm{mp} 132-136{ }^{\circ} \mathrm{C} ;{ }^{1} \mathrm{H} \mathrm{NMR}\left(\mathrm{CDCl}_{3}, 500 \mathrm{MHz}\right): \delta=7.21$ 7.16, 7.11-7.05 (2 m, 2 H each, Ar), 6.53 (dd, $J=3.0,9.8 \mathrm{~Hz}, 1 \mathrm{H}, 3-\mathrm{H}), 5.61$ (br. d, $J \cdot 9.8$ $\mathrm{Hz}, 1 \mathrm{H}, 4-\mathrm{H}), 3.29-3.24$ (m, $1 \mathrm{H}, 2-\mathrm{H}), 3.08$ (ddd, $J=6.2,6.3,13.0 \mathrm{~Hz}, 1 \mathrm{H}, 11-\mathrm{H}), 2.68$ (m, $1 \mathrm{H}, 13-\mathrm{H}), 2.35-2.30$ (m, $1 \mathrm{H}, 16-\mathrm{H}), 2.13$ (quint., $J=6.9 \mathrm{~Hz}, 1 \mathrm{H}, 17-\mathrm{H}), 1.90-1.83$ (m, $2 \mathrm{H}$, 12-H, 16-H), 1.58 (dt, $J=2.0,13.0 \mathrm{~Hz}, 1 \mathrm{H}, 12-\mathrm{H}), 1.08,1.05(2 \mathrm{~d}, J=6.9 \mathrm{~Hz}, 3 \mathrm{H}$ each, $\left.\mathrm{CH}_{3}\right) ;{ }^{13} \mathrm{C} \mathrm{NMR}\left(\mathrm{CDCl}_{3}, 126 \mathrm{MHz}\right): \delta=178.5$ (s, C=O), 136.9, 132.1 (2 s, Ar), 128.9, 128.2, 128.0, 127.3, 126.7, 124.6 (6 d, Ar, C-3, C-4), 90.6 (s, C-1), 39.3 (d, C-13), 39.0 (d, C-2), 35.3 (d, C-11), 34.9 (t, C-16), 32.2 (t, C-12), 30.8 (d, C-17), 17.0, 16.4 (2 q, $\mathrm{CH}_{3}$ ); IR (KBr): v $=3040-2850 \mathrm{~cm}^{-1}(=\mathrm{C}-\mathrm{H}, \mathrm{C}-\mathrm{H}), 1775(\mathrm{C}=\mathrm{O}) ; \mathrm{MS}\left(\mathrm{EI}, 180{ }^{\circ} \mathrm{C}, 80 \mathrm{eV}\right): \mathrm{m} / z(\%)=268(60)$ 
$\left[\mathrm{M}^{+}\right], 251(13)\left[\mathrm{M}^{+}-\mathrm{OH}\right], 223(6)\left[\mathrm{M}^{+}-\mathrm{CO}_{2} \mathrm{H}\right], 128(100)\left[\mathrm{C}_{10} \mathrm{H}_{8}^{+}\right] ; \mathrm{C}_{18} \mathrm{H}_{20} \mathrm{O}_{2}$ (268.4) calcd.: $\mathrm{C}$ 80.56, H 7.51; found: C 80.01, H 7.56.

rac-(2a $R, 2 \mathrm{~b} R, 8 \mathrm{~b} R, 10 \mathrm{a} R)-1,2 \mathrm{~b}, 8 \mathrm{~b}, 9,10,10 \mathrm{a}-H e x a h y d r o c y c l o b u t a[a] p h e n a n t h r e n-2 \mathrm{a}(2 H)-$ ol (21)

Analytical data: colorless oil; ${ }^{1} \mathrm{H}$ NMR $\left(\mathrm{CDCl}_{3}, 500 \mathrm{MHz}\right): \delta=7.17-7.13$ (m, $\left.3 \mathrm{H}, \mathrm{Ar}\right), 7.03$ $(\mathrm{d}, J=7.5 \mathrm{~Hz}, 1 \mathrm{H}, \mathrm{Ar}), 6.47$ (dd, $J=2.5,9.6 \mathrm{~Hz}, 1 \mathrm{H}, 4-\mathrm{H}), 6.18(\mathrm{dd}, J=4.9,9.6 \mathrm{~Hz}, 1 \mathrm{H}, 3-$ H), $3.02\left(\mathrm{~m}_{\mathrm{c}}, 1 \mathrm{H}, 8 \mathrm{~b}-\mathrm{H}\right), 2.67$ ( $\left.\mathrm{c}, 1 \mathrm{H}, 2 \mathrm{~b}-\mathrm{H}\right), 2.26\left(\mathrm{~m}_{\mathrm{c}}, 1 \mathrm{H}, 10 \mathrm{a}-\mathrm{H}\right), 2.14-2.06$ (m, $2 \mathrm{H}, 2-\mathrm{H}$, 9-H), 1.91-1.78 (m, 2 H, 1-H, 10-H), 1.74-1.67 (m, 1 H, 2-H), 1.63 (br. s, 1 H, OH), 1.52-1.45 (m, $1 \mathrm{H}, 10-\mathrm{H}), 1.39\left(\mathrm{~m}_{\mathrm{c}}, 1 \mathrm{H}, 9-\mathrm{H}\right), 1.27-1.20(\mathrm{~m}, 1 \mathrm{H}, 1-\mathrm{H}) ;{ }^{13} \mathrm{C} \mathrm{NMR}\left(\mathrm{CDCl}_{3}, 125 \mathrm{MHz}\right): \delta$ $=139.0,133.4$ (2 s, C-4a, C-8a), 128.4 (d, C-3), 128.0 (d, C-4), 127.5, 126.4, 126.3, 126.2 (4 d, Ar), 75.2 (s, C-2a), 43.5 (d, C-2b), 43.3 (d, C-10a), 36.3 (d, C-8b), 33.2 (t, C-2), 24.5 (t, C10), 23.0 (t, C-9), 18.9 (C-1); IR (film): $v=3380 \mathrm{~cm}^{-1}$ (br. s, OH), 3035-2830 (=C-H, C-H), $1630(\mathrm{C}=\mathrm{C}) ; \mathrm{MS}\left(\mathrm{EI}, 70{ }^{\circ} \mathrm{C}, 80 \mathrm{eV}\right): \mathrm{m} / z(\%)=226(20)\left[\mathrm{M}^{+}\right], 196(100)\left[\mathrm{M}^{+}-\mathrm{CH}_{2} \mathrm{O}\right], 181$ (16) $\left[\mathrm{M}^{+}-\mathrm{C}_{2} \mathrm{H}_{5} \mathrm{O}\right]$; HRMS $\left(70{ }^{\circ} \mathrm{C}, 80 \mathrm{eV}\right):\left[\mathrm{M}^{+}\right]: \mathrm{C}_{16} \mathrm{H}_{18} \mathrm{O}$ calcd.: 226.1358; found: 242.1366; $\mathrm{C}_{16} \mathrm{H}_{18} \mathrm{O}$ (226.3) calcd.: C 84.91, H 8.02; found: C 84.71, H 7.97.

rac-(3aR,3bR,9bR,11aS)-1,2,3,3b,9b,10,11,11a-Octahydro-3a $H$ cyclopenta[a]phenanthren-3a-ol (22)

Analytical data: colorless solid; mp 114-116 ${ }^{\circ} \mathrm{C} ;{ }^{1} \mathrm{H} \mathrm{NMR}\left(\mathrm{CDCl}_{3}, 500 \mathrm{MHz}\right): \delta=7.22-7.09$ (m, $3 \mathrm{H}, \mathrm{Ar}), 7.02$ (d, $J=7.7 \mathrm{~Hz}, 1 \mathrm{H}, \mathrm{Ar}), 6.52$ (d, $J=9.5 \mathrm{~Hz}, 1 \mathrm{H}, 5-\mathrm{H}), 6.18$ (dd, $J=5.2$, $9.5 \mathrm{~Hz}, 1 \mathrm{H}, 4-\mathrm{H}), 3.30-3.22$ (m, $1 \mathrm{H}, 9 \mathrm{~b}-\mathrm{H}), 2.65$ (dd, $J=5.2,7.0 \mathrm{~Hz}, 1 \mathrm{H}, 3 \mathrm{~b}-\mathrm{H}), 2.42-2.28$ (m, $1 \mathrm{H}, 10-\mathrm{H}), 2.04-1.89$ (m, $1 \mathrm{H}, 1-\mathrm{H}), 1.80-1.70$ (m, $1 \mathrm{H}, 11 \mathrm{a}-\mathrm{H}), 1.65-1.60$ (m, $1 \mathrm{H}, 10-$ $\mathrm{H}), 1.60-1.55(\mathrm{~m}, 2 \mathrm{H}, 11-\mathrm{H}, 2-\mathrm{H}), 1.55-1.20(\mathrm{~m}, 5 \mathrm{H}, 11-\mathrm{H}, 3-\mathrm{H}, 2-\mathrm{H}, 1-\mathrm{H}) ;{ }^{13} \mathrm{C}$ NMR $\left(\mathrm{CDCl}_{3}, 125 \mathrm{MHz}\right): \delta=137.2,134.4$ (2 s, C-5a, C-9a), 129.9 (d, C-4), 128.5 (d, C-5), 127.4, 126.2, 126.0, 124.1 (4 d, Ar), 84.1 (s, C-3a), 47.6 (d, C-11a), 44.2 (d, C-3b), 36.5 (d, C-9b), 33.2 (t, C-3), 29.1 (t, C-1), 28.0 (t, C-11), 24.4 (t, C-10), 19.8 (t, C-2); IR (KBr): v = 3380 $\mathrm{cm}^{-1}$ (br. s, OH), 3050-2800 (=C-H, C-H); MS (EI, $\left.100{ }^{\circ} \mathrm{C}, 80 \mathrm{eV}\right): \mathrm{m} / z(\%)=240(52)\left[\mathrm{M}^{+}\right]$, 223 (3) $\left[\mathrm{M}^{+}-\mathrm{H}_{2} \mathrm{O}\right], 154$ (49), 129 (62), 128 (100) $\left[\mathrm{C}_{10} \mathrm{H}_{8}^{+}\right]$; HRMS (100 $\left.{ }^{\circ} \mathrm{C}, 80 \mathrm{eV}\right):\left[\mathrm{M}^{+}\right]$: $\mathrm{C}_{17} \mathrm{H}_{20} \mathrm{O}$ calcd.: 240.1514; found: 240.1537; $\mathrm{C}_{17} \mathrm{H}_{20} \mathrm{O}$ (240.4) calcd.: C 84.96, H 8.39; found: 84.67, H 8.25. 


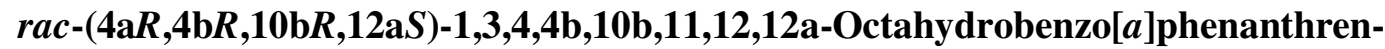
4a(2H)-ol (23)

Analytical data: colorless solid; $\mathrm{mp} 106-108{ }^{\circ} \mathrm{C} ;{ }^{1} \mathrm{H}$ NMR $\left(\mathrm{CDCl}_{3}, 500 \mathrm{MHz}\right): \delta=7.20-7.10$ (m, $3 \mathrm{H}, \mathrm{Ar}), 7.01(\mathrm{~d}, J=7.5 \mathrm{~Hz}, 1 \mathrm{H}, \mathrm{Ar}), 6.51(\mathrm{~d}, J=9.8 \mathrm{~Hz}, 1 \mathrm{H}, 6-\mathrm{H}), 6.18$ (dd, $J=5.7$, $9.8 \mathrm{~Hz}, 1 \mathrm{H}, 5-\mathrm{H}), 3.25$ (m $, 1 \mathrm{H}, 10 \mathrm{~b}-\mathrm{H}), 2.63$ (dd, $J=5.7,7.0 \mathrm{~Hz}, 1 \mathrm{H}, 4 \mathrm{~b}-\mathrm{H}), 2.37-2.30$ (m, $1 \mathrm{H}, 11-\mathrm{H}), 2.00-1.90\left(\mathrm{~m}, 1 \mathrm{H}, \mathrm{CH}_{2}\right), 1.79-1.72(\mathrm{~m}, 1 \mathrm{H}, 12 \mathrm{a}-\mathrm{H}), 1.68-1.54\left(\mathrm{~m}, 5 \mathrm{H}, \mathrm{CH}_{2}\right.$, $\mathrm{OH}), 1.46-1.20\left(\mathrm{~m}, 6 \mathrm{H}, \mathrm{CH}_{2}\right) ;{ }^{13} \mathrm{C} \mathrm{NMR}\left(\mathrm{CDCl}_{3}, 125 \mathrm{MHz}\right): \delta=137.2,134.4$ (2 s, Ar), 129.9 (d, C-5), 128.4 (d, C-6), 127.3, 126.2, 125.9, 124.1 (4 d, Ar), 84.0 (s, C-4a), 47.5 (d, C-12a), 44.1 (d, C-4b), 36.5 (d, C-10b), 33.2, 29.0, 26.4, 24.4, 19.8, 19.7 (6 t, $\left.\mathrm{CH}_{2}\right)$; IR (KBr): v = $3365 \mathrm{~cm}^{-1}$ (br. s, OH), 3000-2800 (=C-H, C-H); MS (EI, $\left.80{ }^{\circ} \mathrm{C}, 80 \mathrm{eV}\right): \mathrm{m} / z(\%)=254(1)$ $\left[\mathrm{M}^{+}\right], 222(33)\left[\mathrm{M}^{+}-\mathrm{CH}_{3} \mathrm{OH}\right], 154(88), 128(100)\left[\mathrm{C}_{10} \mathrm{H}_{8}^{+}\right] ; \mathrm{C}_{18} \mathrm{H}_{22} \mathrm{O}$ (254.4) calcd.: $\mathrm{C}$ 84.99, H 8.72; found: C 84.90, H 8.58.

rac-(4bR,6aS,11aR,11bR)-4b,5,6,6a,7,8,9,10,11,11b-Decahydro-11aHcyclohepta $[a]$ phenanthren-11a-ol (24)

Analytical data: colorless solid; $\mathrm{mp} 109-111{ }^{\circ} \mathrm{C} ;{ }^{1} \mathrm{H} \mathrm{NMR}\left(\mathrm{CDCl}_{3}, 500 \mathrm{MHz}\right): \delta=7.16-7.08$ (m, 3 H, Ar), 7.03-6.98 (m, 1 H, Ar), 6.45 (dd, $J=2.7,9.9$ Hz, 1 H, 13-H), 6.06 (dd, $J=3.3$, $9.9 \mathrm{~Hz}, 1 \mathrm{H}, 12-\mathrm{H}), 3.17$ ( $\left.\mathrm{m}_{\mathrm{c}}, 1 \mathrm{H}, 4 \mathrm{~b}-\mathrm{H}\right), 2.53$ ( $\left.\mathrm{m}_{\mathrm{c}}, 1 \mathrm{H}, 11 \mathrm{~b}-\mathrm{H}\right), 1.97-1.15$ (m, $16 \mathrm{H}, \mathrm{CH}$, $\left.\mathrm{CH}_{2}, \mathrm{OH}\right) ;{ }^{13} \mathrm{C} \mathrm{NMR}\left(\mathrm{CDCl}_{3}, 125 \mathrm{MHz}\right): \delta=139.9,133.1$ (2 s, Ar), 129.0, 127.8, 127.4, 126.2*, 125.9 (5 d, Ar, C-12, C-13), 76.5 (s, C-11a), 46.5 (d, C-6a), 44.6 (d, C-11b), 37.3 (d, C-4b), 33.3, 29.8, 29.5, 29.1, 24.4, 20.8, 19.7 (7 t, $\left.\mathrm{CH}_{2}\right)$ *signal with higher intensity; IR $(\mathrm{KBr}): \mathrm{v}=3435 \mathrm{~cm}^{-1}$ (br. s, OH), 3060-2800 (=C-H, C-H); MS (EI, $\left.90{ }^{\circ} \mathrm{C}, 80 \mathrm{eV}\right): \mathrm{m} / \mathrm{z}(\%)=$ $268(38)\left[\mathrm{M}^{+}\right], 250(16)\left[\mathrm{M}^{+}-\mathrm{H}_{2} \mathrm{O}\right], 154$ (100), 128 (49) $\left[\mathrm{C}_{10} \mathrm{H}_{8}^{+}\right]$; HRMS (90 $\left.{ }^{\circ} \mathrm{C}, 80 \mathrm{eV}\right)$ : $\left[\mathrm{M}^{+}\right]: \mathrm{C}_{19} \mathrm{H}_{24} \mathrm{O}$ calcd.: 268.1827; found: 268.1842; $\mathrm{C}_{19} \mathrm{H}_{24} \mathrm{O}$ (268.4) calcd.: C 85.03, H 9.01; found: C 84.90, H 8.58 .

rac-(4bR,6a $S, 11 \mathrm{a} R, 12 \mathrm{~b} R)-4 \mathrm{~b}, 6,6 \mathrm{a}, 7,8,9,10,11,12,12 \mathrm{~b}-$

Decahydrocycloocta $[a]$ phenanthren-12(5H)-ol (25)

Analytical data: colorless oil; (purity • $90 \%$ ); ${ }^{1} \mathrm{H} \mathrm{NMR}\left(\mathrm{CDCl}_{3}, 500 \mathrm{MHz}\right): \delta=7.26$ (br. d, $J \cdot 7.5 \mathrm{~Hz}, 1 \mathrm{H}, \mathrm{Ar}), 7.16$ (dt, $J=1.3,7.5 \mathrm{~Hz}, 1 \mathrm{H}, \mathrm{Ar}), 7.09$ (tt, $J=1.3,7.3 \mathrm{~Hz}, 1 \mathrm{H}, \mathrm{Ar}$ ), 6.99 $(\mathrm{dd}, J=1.3,7.3 \mathrm{~Hz}, 1 \mathrm{H}, \mathrm{Ar}), 6.56$ (d, $J=9.8 \mathrm{~Hz}, 1 \mathrm{H}, 14-\mathrm{H}), 6.12$ (dd, $J=6.0,9.8 \mathrm{~Hz}, 1 \mathrm{H}$, 13-H), 3.19 ( $\left.\mathrm{m}_{\mathrm{c}}, 1 \mathrm{H}, 4 \mathrm{~b}-\mathrm{H}\right), 2.56-2.50\left(\mathrm{~m}, 2 \mathrm{H}, \mathrm{CH}_{2}\right), 2.38$ ( $\left.\mathrm{m}_{\mathrm{c}}, 1 \mathrm{H}, 12 \mathrm{~b}-\mathrm{H}\right), 1.85-0.86$ (m, 16 $\left.\mathrm{H}, \mathrm{CH}, \mathrm{CH}_{2}, \mathrm{OH}\right) ;{ }^{13} \mathrm{C} \mathrm{NMR}\left(\mathrm{CDCl}_{3}, 125 \mathrm{MHz}\right): \delta=138.9,135.4$ (2 s, Ar), 131.2, 129.8, 
127.5, 125.9, 125.5, 122.9 (6 d, Ar, C-13, C-14), 63.1 (s, C-12a), 42.8 (d, C-6a), 42.0 (d, C12b), 35.6 (d, C-4b), 32.3, 30.0, 29.6, 28.4, 26.6, 26.2, 26.0, 23.1 (8 t, $\mathrm{CH}_{2}$ ); IR (film): $v=$ $3435 \mathrm{~cm}^{-1}$ (br. s, OH), 3025-2850 (=C-H, C-H); MS (EI, $\left.130{ }^{\circ} \mathrm{C}, 80 \mathrm{eV}\right): \mathrm{m} / z(\%)=(6)\left[\mathrm{M}^{+}-\right.$ $\left.\mathrm{H}_{2} \mathrm{O}\right], 207$ (100), $128(70)\left[\mathrm{C}_{10} \mathrm{H}_{8}^{+}\right]$.

rac-(3a $R, 3 \mathrm{~b} R, 9 \mathrm{~b} R, 11 \mathrm{a} S)-1,2,3,3 \mathrm{~b}, 4,5,9 \mathrm{~b}, 10,11,11 \mathrm{a}-D e c a h y d r o-3 \mathrm{a} H$ cyclopenta $[a]$ phenanthren-3a-ol (26)

$0.240 \mathrm{~g}(1.00 \mathrm{mmol}) 22$ and $0.100 \mathrm{~g} \mathrm{Pd}(10 \%) / \mathrm{C}$ in $12 \mathrm{~mL}$ methanol were stirred for $24 \mathrm{~h}$ under hydrogen atmosphere. Filtration on celite and evaporation gave $0.238 \mathrm{~g}$ (98 \%) of 26.

Analytical data: colorless solid; mp 108-111 ${ }^{\circ} \mathrm{C} ;{ }^{1} \mathrm{H}$ NMR $\left(\mathrm{CDCl}_{3}, 270 \mathrm{MHz}\right): \delta=7.24-7.09$ (m, $4 \mathrm{H}, \mathrm{Ar}), 3.14$ (m, $1 \mathrm{H}, 9 \mathrm{~b}-\mathrm{H}), 2.96-2.74$ (m, $3 \mathrm{H}, 11 \mathrm{a}-\mathrm{H}, 5-\mathrm{H}), 2.19-1.48$ (m, $14 \mathrm{H}, \mathrm{CH}$, $\left.\mathrm{CH}_{2}, \mathrm{OH}\right) ;{ }^{13} \mathrm{C} \mathrm{NMR}\left(\mathrm{CDCl}_{3}, 67.9 \mathrm{MHz}\right): \delta=141.2,137.1$ (2 s, Ar), 128.4, 127.7, 125.8, 125.6 (4 d, Ar), 82.6 (s, C-3a), 46.1, 44.2, 37.2 (3 d, CH), 36.8, 30.0, 28.3, 26.7, 25.2, 22.0, $20.5\left(7 \mathrm{t}, \mathrm{CH}_{2}\right)$; IR (KBr): $v=3380 \mathrm{~cm}^{-1}$ (br. s, OH), 3050-2800 (=C-H, C-H); MS (EI, $70{ }^{\circ} \mathrm{C}$, $80 \mathrm{eV}): m / z(\%)=242(45)\left[\mathrm{M}^{+}\right], 224(100)\left[\mathrm{M}^{+}-\mathrm{H}_{2} \mathrm{O}\right], 200(26)\left[\mathrm{M}^{+}-\mathrm{C}_{2} \mathrm{H}_{2} \mathrm{O}\right] ; \mathrm{HRMS}\left(70{ }^{\circ} \mathrm{C}\right.$, $80 \mathrm{eV}):\left[\mathrm{M}^{+}\right]: \mathrm{C}_{17} \mathrm{H}_{22} \mathrm{O}$ calcd.: 242.1670; found: 242.1666 .

\section{rac-(3aR,11aS)-1,2,3,10,11,11a-Hexahydro-3a $H$-cyclopenta[a]phenanthren-3a-ol (27)}

A suspension of $0.100 \mathrm{~g}(0.420 \mathrm{mmol}) 22$ and $0.869 \mathrm{~g}(10.0 \mathrm{mmol}) \mathrm{MnO}_{2}$ in $25 \mathrm{~mL} \mathrm{THF}$ was refluxed. Conversion was monitored by TLC. After $2 \mathrm{~h} 0.869 \mathrm{~g}(10.0 \mathrm{mmol}) \mathrm{MnO}_{2}$ were added, the suspension refluxed for again $2 \mathrm{~h}$. After cooling to room temperature the mixture was filtrated on celite, evaporated and diluted with $\mathrm{CH}_{2} \mathrm{Cl}_{2}$. The solution was washed with $1 \mathrm{~N}$ $\mathrm{HCl}$ and water, dried over anhydrous magnesium sulfate, filtered and evaporated. Chromatography of the resulting crude product on silica gel using hexane/ethyl acetate mixture (90:10) afforded $0.073 \mathrm{~g}(73 \%)$ of 27.

Analytical data: colorless solid; $\mathrm{mp} 93-95{ }^{\circ} \mathrm{C} ;{ }^{1} \mathrm{H} \mathrm{NMR}\left(\mathrm{CDCl}_{3}, 270 \mathrm{MHz}\right): \delta=7.97,7.79$ (2 d, $J=8.1 \mathrm{~Hz}, 1 \mathrm{H}$ each, Ar), 7.73-7.63, 7.54-7.42 (2 m, $2 \mathrm{H}$ each, Ar), $3.72\left(\mathrm{~m}_{\mathrm{c}}, 1 \mathrm{H}, 11 \mathrm{a}-\right.$ H), $3.31(\mathrm{td}, J=4.6,16.7 \mathrm{~Hz}, 1 \mathrm{H}, 10-\mathrm{H}), 2.97$ (ddd, $J=5.5,10.7,16.7 \mathrm{~Hz}, 1 \mathrm{H}, 10-\mathrm{H}), 2.35-$ $1.22\left(\mathrm{~m}, 9 \mathrm{H}, \mathrm{CH}_{2}, \mathrm{OH}\right) ;{ }^{13} \mathrm{C} \mathrm{NMR}\left(\mathrm{CDCl}_{3}, 67.9 \mathrm{MHz}\right): \delta=138.7,132.4,131.5,131.2(4 \mathrm{~s}$, Ar), 128.3, 126.7, 126.0, 125.4*, 123.7 (5 d, Ar), 82.2 (s, C-3a), 47.9 (d, C-11a), 40.5, 30.3, 28.4, 24.8, $22.1\left(5 \mathrm{t}, \mathrm{CH}_{2}\right)$ *signal with higher intensity; IR (KBr): v $=3380 \mathrm{~cm}^{-1}$ (br. s, OH), 3050-2800 (=C-H, C-H).; MS (EI, $\left.70{ }^{\circ} \mathrm{C}, 80 \mathrm{eV}\right): \mathrm{m} / \mathrm{z}(\%)=238(100)\left[\mathrm{M}^{+}\right], 209$ (99) $\left[\mathrm{M}^{+}-\right.$ 
$\left.\mathrm{C}_{2} \mathrm{H}_{5}\right], 196$ (90) $\left[\mathrm{M}^{+}-\mathrm{C}_{2} \mathrm{H}_{2} \mathrm{O}\right.$ ]; $\mathrm{C}_{17} \mathrm{H}_{18} \mathrm{O}$ (238.3) calcd.: C 85.67, H 7.61; found: $\mathrm{C}$ 85.15, H 7.47 .

rac-(1aS,5bR,7aS,10a $R, 10 \mathrm{~b} S, 10 \mathrm{c} R)-1 \mathrm{a}, 5 \mathrm{~b}, 6,7,7 \mathrm{a}, 8,9,10,10 \mathrm{~b}, 10 \mathrm{c}-D e c a h y d r o-10 \mathrm{a} H$ cyclopenta[1,2]phenanthro[9,10-b]oxiren-10a-ol (28)

A solution of $0.240 \mathrm{~g}(1.00 \mathrm{mmol}) 22$ and $0.257 \mathrm{~g}(1.05 \mathrm{mmol}) \mathrm{m}$-CPBA (70\%) in $1.5 \mathrm{~mL}$ $\mathrm{CH}_{2} \mathrm{Cl}_{2}$ was stirred first at $0{ }^{\circ} \mathrm{C}$, then at room temperature. The conversion was monitored by TLC. After $8 \mathrm{~h}$ the solution was diluted with $10 \mathrm{~mL} \mathrm{CH}_{2} \mathrm{Cl}_{2}$, washed with $4 \mathrm{~N} \mathrm{NaOH}$ solution and water. The organic layer was dried over anhydrous magnesium sulfate, filtered and evaporated to afford $0.256 \mathrm{~g}$ (quant.) 28.

Analytical data: colorless solid; mp 109-110 ${ }^{\circ} \mathrm{C} ;{ }^{1} \mathrm{H}$ NMR $\left(\mathrm{CDCl}_{3}, 500 \mathrm{MHz}\right): \delta=7.37$ (d, $J$ = 7.4 Hz, $1 \mathrm{H}, \mathrm{Ar}), 7.31-7.24$ (m, $1 \mathrm{H}, \mathrm{Ar}), 7.23-7.15$ (m, $2 \mathrm{H}, \mathrm{Ar}), 4.00$ (dd, J = 2.6, $4.3 \mathrm{~Hz}, 1$ H, 10c-H), 3.85 (d, $J=4.3 \mathrm{~Hz}, 1 \mathrm{H}, 1 \mathrm{a}-\mathrm{H}), 3.17-3.10$ (m, $1 \mathrm{H}, 5 \mathrm{~b}-\mathrm{H}), 2.75$ (dd, $J=2.6,7.0$ $\mathrm{Hz}, 1 \mathrm{H}, 10 \mathrm{~b}-\mathrm{H}), 2.45$ (qd, $J=3.2,14.0 \mathrm{~Hz}, 1 \mathrm{H}, 6-\mathrm{H}), 2.01-1.91$ (m, $1 \mathrm{H}, 8-\mathrm{H}), 1.79-0.94$ (m, $8 \mathrm{H}, 6-\mathrm{H}, 7-\mathrm{H}, 7 \mathrm{a}-\mathrm{H}, 10-\mathrm{H}, 9-\mathrm{H}, 8-\mathrm{H}, \mathrm{OH}), 0.82-0.69$ (m, $1 \mathrm{H}, 9-\mathrm{H}) ;{ }^{13} \mathrm{C} \mathrm{NMR}\left(\mathrm{CDCl}_{3}, 126\right.$ MHz): $\delta=137.0,133.1$ ( 2 s, Ar), 129.7, 128.8, 125.6, 124.3 (4 d, Ar), 82.9 (s, C-10a), 55.6 (d, C-10c), 53.9 (d, C-1a), 49.5 (d, C-7a), 42.8 (d, C-10b), 31.8 (t, C-9), 31.1 (d, C-5b), 29.5 (t, C-8), 28.3 (t, C-7), 24.6 (t, C-6), 20.0 (t, C-10); IR (KBr): v = $3365 \mathrm{~cm}^{-1}$ (br. s, OH), $3065-$ 2850 (=C-H, C-H); MS (EI, $\left.70{ }^{\circ} \mathrm{C}, 80 \mathrm{eV}\right): \mathrm{m} / z(\%)=256(16)\left[\mathrm{M}^{+}\right], 238(16)\left[\mathrm{M}^{+}-\mathrm{H}_{2} \mathrm{O}\right], 154$ (35), 145 (41), 86 (44), 84 (66), 49 (100); $\mathrm{C}_{17} \mathrm{H}_{20} \mathrm{O}_{2}$ (256.4) calcd.: C 79.65, H 7.86; found: C $79.88, \mathrm{H} 7.84$.

rac-(3aS,3bR,9bR,11S,11aS)-Methyl 3a-Hydroxy-2,3,3a,3b,9b,10,11,11a-octahydro-1Hcyclopenta $[a]$ phenanthren-11-carboxylate (30)

Analytical data: foamy resin; ${ }^{1} \mathrm{H}$ NMR $\left(\mathrm{CDCl}_{3}, 500 \mathrm{MHz}\right): \delta=7.14\left(\mathrm{~m}_{\mathrm{c}}, 2 \mathrm{H}, 8-\mathrm{H}, 7-\mathrm{H}\right)$, $7.08\left(\mathrm{~m}_{\mathrm{c}}, 1 \mathrm{H}, 9-\mathrm{H}\right), 7.01$ (m $\left., 1 \mathrm{H}, 6-\mathrm{H}\right), 6.42$ (br. d, $\left.J \bullet 9.7 \mathrm{~Hz}, 1 \mathrm{H}, 5-\mathrm{H}\right), 5.74$ (d, J = 9.7 Hz, $1 \mathrm{H}, 4-\mathrm{H}), 3.61$ (s, $\left.3 \mathrm{H}, \mathrm{OCH}_{3}\right), 3.13-3.07$ (m, $\left.1 \mathrm{H}, 9 \mathrm{~b}-\mathrm{H}\right), 2.96(\mathrm{td}, J=5.2,13.4 \mathrm{~Hz}, 1 \mathrm{H}, 11-$ H), 2.69 (br. s, $1 \mathrm{H}, 3 \mathrm{~b}-\mathrm{H}), 2.36\left(\mathrm{~m}_{\mathrm{c}}, 1 \mathrm{H}, 11 \mathrm{a}-\mathrm{H}\right), 2.15\left(\mathrm{~m}_{\mathrm{c}}, 1 \mathrm{H}, 3-\mathrm{H}_{\mathrm{B}}\right), 1.98(\mathrm{q}, J=13.4 \mathrm{~Hz}, 1$ $\left.\mathrm{H}, 10-\mathrm{H}_{\mathrm{B}}\right), 1.92-1.49\left(\mathrm{~m}, 6 \mathrm{H}, 2-\mathrm{H}, 3-\mathrm{H}_{\mathrm{A}}, 1-\mathrm{H}, \mathrm{OH}\right), 1.46\left(\mathrm{td}, J=3.4,13.4 \mathrm{~Hz}, 1 \mathrm{H}, 10-\mathrm{H}_{\mathrm{A}}\right)$; ${ }^{13} \mathrm{C}$ NMR $\left(\mathrm{CDCl}_{3}, 126 \mathrm{MHz}\right): \delta=175.3$ (s, C=O), 139.3, 132.4 (2 s, C-5a, C-9a), 127.7, 127.5*, 127.2 (3 d, C-8, C-7, C-5, C-4), 126.8 (d, C-9), 126.1 (d, C-6), 81.2 (s, C-3a), 51.5 (q, $\mathrm{OCH}_{3}$ ), 47.0 (d, C-11a), 44.6 (d, C-3b), 39.4 (d, C-11), 38.0 (t, C-3), 36.9 (d, C-9b), 25.0 (t, C-10), 24.1 (t, C-1), 20.8 (t, C-2) *signal with higher intensity; IR (KBr): v = $3440 \mathrm{~cm}^{-1}$ (br. s, 
$\mathrm{OH}), 3100-2900(=\mathrm{C}-\mathrm{H}, \mathrm{C}-\mathrm{H}), 1735(\mathrm{C}=\mathrm{O}), 1630(\mathrm{C}=\mathrm{C})$; MS (EI, $\left.120^{\circ} \mathrm{C}, 80 \mathrm{eV}\right): \mathrm{m} / \mathrm{z}(\%)=$ 298 (37) $\left[\mathrm{M}^{+}\right], 280(22)\left[\mathrm{M}^{+}-\mathrm{H}_{2} \mathrm{O}\right], 220(10)\left[\mathrm{M}^{+}-\mathrm{H}_{2} \mathrm{O}-\mathrm{HCO}_{2} \mathrm{CH}_{3}\right]$; HRMS $\left(120{ }^{\circ} \mathrm{C}, 80 \mathrm{eV}\right)$ : $\left[\mathrm{M}^{+}\right]: \mathrm{C}_{19} \mathrm{H}_{22} \mathrm{O}_{3}$ calcd.: 298.1569; found: 298.1538 .

rac-(3aS,3bR,9bR,11R,11aS)- Methyl 3a-Hydroxy-2,3,3a,3b,9b,10,11,11a-octahydro-1Hcyclopenta[a]phenanthren-11-carboxylate (32)

Analytical data: foamy resin; ${ }^{1} \mathrm{H}$ NMR $\left(\mathrm{CDCl}_{3}, 500 \mathrm{MHz}\right): \delta=7.22-7.10(\mathrm{~m}, 3 \mathrm{H}, 7-\mathrm{H}, 8-\mathrm{H}$, 9-H), 7.02 (d, $J=6.5 \mathrm{~Hz}, 1 \mathrm{H}, 6-\mathrm{H}), 6.51$ (br. d, $J \bullet 9.8 \mathrm{~Hz}, 1 \mathrm{H}, 5-\mathrm{H}), 6.17$ (dd, $J=5.1,9.8$ $\mathrm{Hz}, 1 \mathrm{H}, 4-\mathrm{H}), 3.72$ (s, $\left.3 \mathrm{H}, \mathrm{OCH}_{3}\right), 3.36$ (td, $\left.J=4.0,7.7 \mathrm{~Hz}, 1 \mathrm{H}, 9 \mathrm{~b}-\mathrm{H}\right), 2.69\left(\mathrm{~m}_{\mathrm{c}}, 1 \mathrm{H}, 3 \mathrm{~b}-\mathrm{H}\right)$, 2.54 (td, $J=4.0,14.0 \mathrm{~Hz}, 1 \mathrm{H}, 10-\mathrm{H}), 2.41$ (br. s, $1 \mathrm{H}, \mathrm{OH}), 2.35$ (dt, $J=4.0,10.6 \mathrm{~Hz}, 1 \mathrm{H}$, 11-H), 2.13-1.81 (m, 3 H, 1-H, 10-H, 11a-H), 1.68-1.54 (m, 1 H, 2-H), 1.50-1.19 (m, 4 H, 1$\mathrm{H}, 2-\mathrm{H}, 3-\mathrm{H}) ;{ }^{13} \mathrm{C} \mathrm{NMR}\left(\mathrm{CDCl}_{3}, 126 \mathrm{MHz}\right): \delta=177.7$ (s, C=O), 136.0, 134.1 (2 s, C-5a, C9a), 129.4, 128.5, 127.6, 126.3, 126.3, 123.9 (6 d, C-4, C-5, C-6, C-7, C-8, C-9), 80.7 (s, C3a), 51.7 (q, $\left.\mathrm{OCH}_{3}\right), 48.7$ (d, C-11a), 43.8 (d, C-3b), 42.3 (d, C-11), 35.3 (t, C-3), 33.5 (d, C9b), 27.8 (t, C-10), 27.8 (t, C-1), 19.6 (t, C-2); IR (KBr): v = $3435 \mathrm{~cm}^{-1}$ (br. s, OH), 3055-2850 (=C-H, C-H), $1730(\mathrm{C}=\mathrm{O}), 1630(\mathrm{C}=\mathrm{C}) ; \mathrm{MS}\left(\mathrm{EI}, 120^{\circ} \mathrm{C}, 80 \mathrm{eV}\right): m / z(\%)=298(8)\left[\mathrm{M}^{+}\right], 280$ (12) $\left[\mathrm{M}^{+}-\mathrm{H}_{2} \mathrm{O}\right], 220$ (6) $\left[\mathrm{M}^{+}-\mathrm{H}_{2} \mathrm{O}-\mathrm{HCO}_{2} \mathrm{CH}_{3}\right], 128$ (17) $\left[\mathrm{C}_{10} \mathrm{H}_{8}^{+}\right] ; \mathrm{C}_{19} \mathrm{H}_{22} \mathrm{O}_{3}$ (298.4) calcd.: $\mathrm{C}$ 76.48, H 7.43; found: C 76.62, H 7.49.

rac-(4bR,6R,6aS,10a $R, 10 \mathrm{~b} R)-$ Methyl 10a-Hydroxy-4b,5,6,6a,7,8,9,10,10a,10bdecahydrobenzo $[a]$ phenanthren-6-carboxylate (34)

Analytical data: colorless solid; $\mathrm{mp}=163-165{ }^{\circ} \mathrm{C} ;{ }^{1} \mathrm{H} \mathrm{NMR}\left(\mathrm{CDCl}_{3}, 500 \mathrm{MHz}\right): \delta=7.25$ 7.21, 7.18-7.15 (2 m, 3 H, Ar, 7.03 (d, J = 7.1 Hz, $1 \mathrm{H}, \mathrm{Ar}), 6.63$ (d, J = 9.6 Hz, $1 \mathrm{H}, 12-\mathrm{H})$, $6.15(\mathrm{dd}, J=6.0,9.6 \mathrm{~Hz}, 1 \mathrm{H}, 11-\mathrm{H}), 3.74\left(\mathrm{~s}, 3 \mathrm{H}, \mathrm{OCH}_{3}\right), 3.41-3.38$ (m, $\left.1 \mathrm{H}, 4 \mathrm{~b}-\mathrm{H}\right), 2.83$ (ddd, $J=2.5,9.3,12.9 \mathrm{~Hz}, 1 \mathrm{H}, 6-\mathrm{H}), 2.64$ (td, $J=2.5,14 \mathrm{~Hz}, 1 \mathrm{H}, 5-\mathrm{H}), 2.50$ (dd, $J=6.0$, $7.4 \mathrm{~Hz}, 1 \mathrm{H}, 10 \mathrm{~b}-\mathrm{H}), 2.06$ (ddd, $J=5.5,12.9,14.0 \mathrm{~Hz}, 1 \mathrm{H}, 5-\mathrm{H}), 1.58$ (br.s, $1 \mathrm{H}, \mathrm{OH}), 1.88$ -

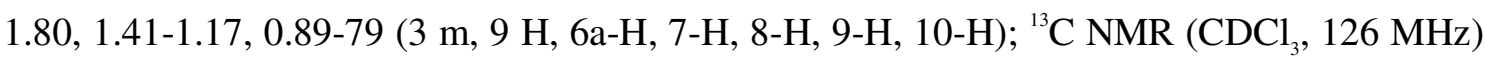
$\delta=175.7$ (s, CO), 136.7, 134.8 (2 s, Ar), 130.3 (d, C-12), 129.1 (d, C-11), 127.9, 126.6, 126.3, 122.9 (4 d, Ar), 73.9 (s, C-10a), 51.7 (q, OCH $), 47.6$ (d, C-10b), 45.6 (d, C-6a), 40.6 (d, C-6), 36.1 (d, C-4b), 28.9 (t, C-5), 27.7, 24.4, 21.2, 20.3 (4 t, C-7, C8, C-9, C-10); IR $(\mathrm{KBr}): v=3510 \mathrm{~cm}^{-1}(\mathrm{O}-\mathrm{H}), 2950-2865(=\mathrm{C}-\mathrm{H}, \mathrm{C}-\mathrm{H}), 1720(\mathrm{C}=\mathrm{O})$; MS (EI, $\left.80 \mathrm{eV}, 40{ }^{\circ} \mathrm{C}\right)$ $\mathrm{m} / \mathrm{z}(\%)=312(22)\left[\mathrm{M}^{+}\right], 294(100)\left[\mathrm{M}^{+}-\mathrm{H}_{2} \mathrm{O}\right], 280(12)\left[\mathrm{M}^{+}-\mathrm{CH}_{3} \mathrm{O} / \mathrm{H}\right], 141$ (56) $\left[\mathrm{C}_{11} \mathrm{H}_{9}^{+}\right]$, 
128 (59) $\left[\mathrm{C}_{10} \mathrm{H}_{8}^{+}\right], 98$ (94), 54 (19); HRMS (80 eV, $\left.40{ }^{\circ} \mathrm{C}\right):\left[\mathrm{M}^{+}\right]: \mathrm{C}_{20} \mathrm{H}_{24} \mathrm{O}_{3}$ calcd.: 312.1725; found 312.1742 .

rac-(1R,2R/S,11R/S,13S,14S)-18-Oxapentacyclo[11.5.2.0 $\left.{ }^{1,14} .0^{2,11} .0^{5,10}\right] \mathrm{icosa}-3,5,7,9-$ tetraen-20-one (35)

Analytical data: colorless solid; $\mathrm{mp}=108-110{ }^{\circ} \mathrm{C} ;{ }^{1} \mathrm{H} \mathrm{NMR}\left(\mathrm{CDCl}_{3}, 500 \mathrm{MHz}\right): \delta=7.16-$ 7.15, 7-06-7.04, 7.02-7.00 (3 m, 4 H, Ar), 6.38 (dd, $J=3.3,10.2 \mathrm{~Hz}, 1 \mathrm{H}, 4-\mathrm{H}), 6.18$ (br. d, $J$ $=10.2 \mathrm{~Hz}, 1 \mathrm{H}, 3-\mathrm{H}), 3.27-3.26(\mathrm{~m}, 1 \mathrm{H}, 2-\mathrm{H}), 3.24-3.19(\mathrm{~m}, 1 \mathrm{H}, 11-\mathrm{H}), 2.68(\mathrm{t}, J=5.0 \mathrm{~Hz}, 1$ H, 13-H), 2.20-2.12 (m, 1 H, 14-H), 2.52-2.22, 2.10-2.04, 2.02-1.95, 1.61-1.55 (4 m, 8 H, 15H, 16-H, 17-H, 18-H), 1.80 (ddd, $J=1.1,13.2,14.3 \mathrm{~Hz}, 1 \mathrm{H}, 12-\mathrm{H}), 1.69$ (td, $J=5.5,13.2$ $\mathrm{Hz}, 12-\mathrm{H}) ;{ }^{13} \mathrm{C}$ NMR $\left(\mathrm{CDCl}_{3}, 126 \mathrm{MHz}\right): \bullet \delta=178.8$ (s, CO), 136.8, 131.5 (2 s, Ar), 128.1, 128.1, 127.3, 126.4 (4 d, Ar), 126.9, 126.3 (2 d, C-3, C-4), 87.2 (s, C-1), 49.8 (d, C-14), 43.5 (d, C-2), 43.1 (d, C-13), 35.7 (d, C-11), 26.7 (t, C-12), 34.9, 24.9, 22.8, 20.5 (4 t, C-15, C-16, C-17, C-18); IR (KBr): v = 2920-2855 (=C-H, C-H), 1775 (C=O), 1490-1450 (C-H); MS (EI, $\left.80 \mathrm{eV}, 100{ }^{\circ} \mathrm{C}\right) \mathrm{m} / \mathrm{z}(\%)=280(56)\left[\mathrm{M}^{+}\right], 234(10), 182(61), 165$ (22) $\left[\mathrm{C}_{13} \mathrm{H}_{9}^{+}\right], 141$ (48) $\left[\mathrm{C}_{11} \mathrm{H}_{9}^{+}\right], 128(100)\left[\mathrm{C}_{10} \mathrm{H}_{8}^{+}\right], 98(12) ; \operatorname{HRMS}\left(80 \mathrm{eV}, 100{ }^{\circ} \mathrm{C}\right):\left[\mathrm{M}^{+}\right]: \mathrm{C}_{19} \mathrm{H}_{20} \mathrm{O}_{2}$ calcd.: 280.1463; found: 280.1457 .

\section{rac-(4bR,6R,6aS,10aR,10bR)-Methyl 8-Ethyleneacetal-10a-hydroxy-}

\section{4b,5,6,6a,7,8,9,10a,10b-decahydrobenzo $[a]$ phenanthren-6-carboxylate (37)}

Analytical data: colorless solid; $\mathrm{mp}=173-175{ }^{\circ} \mathrm{C} ;{ }^{1} \mathrm{H} \mathrm{NMR}\left(\mathrm{CDCl}_{3}, 500 \mathrm{MHz}\right): \delta=7.29$ $7.23(2 \mathrm{~m}, 2 \mathrm{H}, \mathrm{Ar}), 7.16$ (t, $J=7.1 \mathrm{~Hz}, 1 \mathrm{H}, \mathrm{Ar}), 7.03$ (t, $J=7.1 \mathrm{~Hz}, 1 \mathrm{H}, \mathrm{Ar}), 6.64$ (d, J = 9.6 $\mathrm{Hz}, 1 \mathrm{H}, 12-\mathrm{H}), 6.15(\mathrm{dd}, J=6.0,9.6 \mathrm{~Hz}, 1 \mathrm{H}, 11-\mathrm{H}), 3.82-3.79,3.78-3.74(2 \mathrm{~m}, 4 \mathrm{H}$, $\mathrm{OCH}_{2} \mathrm{CH}_{2} \mathrm{O}$ ), 3.76 (s, $\left.3 \mathrm{H}, \mathrm{OCH}_{3}\right), 3.40-3.36(\mathrm{~m}, 1 \mathrm{H}, 4 \mathrm{~b}-\mathrm{H}), 3.13$ (ddd, $J=2.5,10.2,13.5 \mathrm{~Hz}$, $1 \mathrm{H}, 6-\mathrm{H}), 2.67-2.64(\mathrm{~m}, 1 \mathrm{H}, 5-\mathrm{H}), 2.53(\mathrm{dd}, J=6.6 \mathrm{~Hz}, 1 \mathrm{H}, 10 \mathrm{~b}-\mathrm{H}), 2.07-2.02$ (m, $2 \mathrm{H}, 6 \mathrm{a}-$ H, 7-H), 1.91 (ddd, $J=5.0,13.5,18.7 \mathrm{~Hz}, 1 \mathrm{H}, 5-\mathrm{H}), 1.75$ (ddd, $J=4.4,13.7,18.1 \mathrm{~Hz}, 1 \mathrm{H}, 9$ H), 1.51-1.45 (m, 2 H, 7-H, 10-H), 1.39 (br. s, 1 H, OH), 1.28-1.25 (m, 1 H, 9-H), 1.09 (ddd, $J=4.1,14.3,18.1 \mathrm{~Hz}, 1 \mathrm{H}, 10-\mathrm{H}) ;{ }^{13} \mathrm{C} \mathrm{NMR}\left(\mathrm{CDCl}_{3}, 126 \mathrm{MHz}\right): \delta=176.1(\mathrm{~s}, \mathrm{CO}), 136.5$, 134.5 (2 s, Ar), 130.6 (d, C-12), 128.3 (d, C-11), 128.3, 126.6, 126.4, 123.2 (4 d, Ar), 108.2 (s, C-8), 73.4 (s, C-10a), 64.5, 63.8 (2 t, $\left.\mathrm{OCH}_{2} \mathrm{CH}_{2} \mathrm{O}\right), 51.7$ (q, $\left.\mathrm{OCH}_{3}\right), 47.2$ (d, C-10b), 45.6 (d, C-6a), 41.6 (d, C-6), 36.2 (d, C-4b), 32.0 (t, C-7), 30.3 (t, C-9), 29.8 (t, C-5), 26.1 (t, C10); IR (KBr): $v=3485 \mathrm{~cm}^{-1}(\mathrm{O}-\mathrm{H}), 2950-2875$ (=C-H, C-H), $1730(\mathrm{C}=\mathrm{O}), 1450-1435(\mathrm{C}-\mathrm{H})$; MS (EI, $\left.80 \mathrm{eV}, 150{ }^{\circ} \mathrm{C}\right): \mathrm{m} / \mathrm{z}(\%)=370(57)\left[\mathrm{M}^{+}\right], 352(49)\left[\mathrm{M}^{+}-\mathrm{H}_{2} \mathrm{O}\right], 212(24), 156(60)$ 
$\left[\mathrm{C}_{8} \mathrm{H}_{12} \mathrm{O}_{3}^{+}\right], 141(69)\left[\mathrm{C}_{11} \mathrm{H}_{9}^{+}\right], 128(88)\left[\mathrm{C}_{10} \mathrm{H}_{8}^{+}\right], 86(100), 55$ (64), 43 (57) $\left[\mathrm{OCH}_{2} \mathrm{CH}^{+}\right], 28$ (41) $\left[\mathrm{CH}_{2} \mathrm{CH}_{2}^{+}\right]$; HRMS (EI, $80 \mathrm{eV}, 150-160{ }^{\circ} \mathrm{C}$ ): $\left[\mathrm{M}^{+}\right]: \mathrm{C}_{22} \mathrm{H}_{26} \mathrm{O}_{5}$ calcd.: 370.1780, found: 370.1765 .

rac- $(1 R, 2 R / S, 11 R / S, 13 S, 14 S)-16-E$ thyleneacetal-19,20-

dioxapentacyclo[11.5.3.0 $\left.{ }^{1,14} \cdot 0^{2,11} \cdot 0^{5,10}\right]$ henicosa-3,5,7,9-tetraen-20-one (38)

Analytical data: colorless solid; $\mathrm{mp}=193-194{ }^{\circ} \mathrm{C} ;{ }^{1} \mathrm{H}$ NMR $\left(\mathrm{CDCl}_{3}, 500 \mathrm{MHz}\right): \delta=7.17$, $7.16(2 \mathrm{~d}, J=5.5 \mathrm{~Hz}, 2 \mathrm{H}, \mathrm{Ar}), 7.05(\mathrm{dd}, J=3.3,5.5 \mathrm{~Hz}, 1 \mathrm{H}, \mathrm{Ar}), 7.01(\mathrm{dd}, J=3.6,5.5 \mathrm{~Hz}, 1$ H, Ar), 6.42 (dd, J=3.3, 10.2 Hz, 1 H, 4-H), 6.12 (br. d, J • 10.2 Hz, 1 H, 3-H), 4.02-3.94 (m, $4 \mathrm{H}, \mathrm{OCH}_{2} \mathrm{CH}_{2} \mathrm{O}$ ), 3.29-3.27 (m, $\left.1 \mathrm{H}, 2-\mathrm{H}\right), 3.25-3.20$ (m, $\left.1 \mathrm{H}, 11-\mathrm{H}\right), 2.70-2.66,2.64-2.63$ (2 m, 2 H, 13-H, 14-H), 2.41 (t, $J=13.2$ Hz, 1 H, 12-H), 2.29-2.15, 2.09-2.04, 1.79-1.70 (3 m, 7 $\mathrm{H}, 12-\mathrm{H}, 15-\mathrm{H}, 17-\mathrm{H}, 18-\mathrm{H}) ;{ }^{13} \mathrm{C}$ NMR $\left(\mathrm{CDCl}_{3}, 126 \mathrm{MHz}\right) \cdot \delta=178.5$ (s, CO), 136.6, $131.4(2$ s, Ar), 128.3, 15.0 (2 s, C-3, C-4), 128.1, 127.9, 127.3, 126.5 (4 d, Ar), 108.9 (s, C-16), 85.9 (s, C-1), 64.8, 64.4 (2 t, $\left.\mathrm{OCH}_{2} \mathrm{CH}_{2} \mathrm{O}\right), 45.4,42.6$ (2 d, C-13, C-14), 43.4, 35.7 (2 d, C-2, C11), 32.9 (t, C-12), 34.1, 31.3, 26.6 (3 t, C-15, C-17, C-18); IR (KBr): v = $3485 \mathrm{~cm}^{-1}(\mathrm{O}-\mathrm{H})$, 3035-2880 (=C-H, C-H), 1775 (C=O), 1460-1430 (C-H); MS (EI, $\left.80 \mathrm{eV}, 150{ }^{\circ} \mathrm{C}\right): \mathrm{m} / \mathrm{z}(\%)=$ 338 (7) $\left[\mathrm{M}^{+}\right], 279$ (2), 183 (34), $156(100)\left[\mathrm{C}_{8} \mathrm{H}_{12} \mathrm{O}_{3}^{+}\right], 141(35)\left[\mathrm{C}_{11} \mathrm{H}_{9}^{+}\right], 128(7)\left[\mathrm{C}_{10} \mathrm{H}_{8}^{+}\right], 86$ (9), 43 (2) $\left[\mathrm{OCH}_{2} \mathrm{CH}^{+}\right]$; HRMS (80 eV, 150-160 $\left.{ }^{\circ} \mathrm{C}\right):\left[\mathrm{M}^{+}\right]: \mathrm{C}_{21} \mathrm{H}_{22} \mathrm{O}_{4}$ calcd.: 338.1517, found: 338.1532 .

rac-(4bR,6S,6aS,10aR,10bR)-Methyl 10a-Hydroxy-4b,5,6,6a,7,8,9,10,10a,10bdecahydrobenzo $[a]$ phenanthren-6-carboxylate $(40)$

Analytical data: colorless solid; $\mathrm{mp}=171-174{ }^{\circ} \mathrm{C} ;{ }^{1} \mathrm{H}$ NMR $\left(\mathrm{CDCl}_{3}, 500 \mathrm{MHz}\right): \delta=7.37(\mathrm{~d}$, $J=7.4 \mathrm{~Hz}, 1 \mathrm{H}, \mathrm{Ar}), 7.23(\mathrm{dt}, J=1.5,7.4 \mathrm{~Hz}, 1 \mathrm{H}, \mathrm{Ar}), 7.19$ (dt, $J=1.1,7.4 \mathrm{~Hz}, 1 \mathrm{H}, \mathrm{Ar})$, $7.07(\mathrm{dd}, J=1.5,7.4 \mathrm{~Hz}, 1 \mathrm{H}, \mathrm{Ar}), 6.62,6.09(2 \mathrm{dd}, \mathrm{J}=3.0,9.9 \mathrm{~Hz}, \mathrm{~J}=2.0,9.9 \mathrm{~Hz} 2 \mathrm{H}, 11-\mathrm{H}$, 12-H), 3.72 (s, $3 \mathrm{H}, \mathrm{OCH}_{3}$ ), 3.34 (td, $\left.J=4.0,13.2 \mathrm{~Hz}, 1 \mathrm{H}, 6-\mathrm{H}\right), 3.05$ (ddd, $J=4.1,12.6,15.2$ $\mathrm{Hz}, 1 \mathrm{H}, 4 \mathrm{~b}-\mathrm{H}), 2.58$ (td, $J=2.0,15.2 \mathrm{~Hz}, 1 \mathrm{H}, 10 \mathrm{~b}-\mathrm{H}), 2.39$ (td, $J=4.0,13.5 \mathrm{~Hz}, 1 \mathrm{H}, 5-\mathrm{H})$, $2.09(\mathrm{td}, J=4.0,12.1 \mathrm{~Hz}, 1 \mathrm{H}, 6 \mathrm{a}-\mathrm{H}), 1.87$ (td, $J=13.2,13.5 \mathrm{~Hz}, 1 \mathrm{H}, 5-\mathrm{H}), 1.56$ (br. s, $1 \mathrm{H}$, $\mathrm{OH}), 2.34-2.31,1.71-1.68,1.65-1.61,1.45-1.17$ (4 m, 8 H, 7-H, 8-H, 9-H, 10-H); ${ }^{13} \mathrm{C}$ NMR $\left(\mathrm{CDCl}_{3}, 126 \mathrm{MHz}\right): \delta=175.8$ (s, CO), 138.7, 133.9 (2 s, Ar), 130.3, 128.1 (2 d, C-11, C-12), 127.6, 126.4, 125.9, 123.9 (4 d, Ar), 72.6 (s, C-10a), 51.7 (q, $\mathrm{OCH}_{3}$ ), 46.1 (d, C-6a), 41.3 (d, C-6), 38.4 (d, C-10b), 35.8 (d, C-4b), 25.7 (t, C-5), 37.5, 24.4, 24.1, 23.3 (4 t, C-7, C-8, C-9, C-10); IR (KBr): v = $3480 \mathrm{~cm}^{-1}(\mathrm{O}-\mathrm{H}), 2930-2855$ (=C-H, C-H), $1715(\mathrm{C}=\mathrm{O}), 1450-1440(\mathrm{C}-$ 
$\mathrm{H}) ; \mathrm{MS}\left(\mathrm{EI}, 80 \mathrm{eV}, 100{ }^{\circ} \mathrm{C}\right): \mathrm{m} / \mathrm{z}(\%)=312(18)\left[\mathrm{M}^{+}\right], 294(55)\left[\mathrm{M}^{+}-\mathrm{H}_{2} \mathrm{O}\right], 280(41)\left[\mathrm{M}^{+}-\right.$ $\left.\mathrm{CH}_{3} \mathrm{O} / \mathrm{H}\right], 234$ (100) $\left[\mathrm{M}^{+}-\mathrm{H}_{2} \mathrm{O} / \mathrm{CH}_{3} \mathrm{O} / \mathrm{H}\right], 183$ (49), 165 (41) $\left[\mathrm{C}_{13} \mathrm{H}_{9}^{+}\right], 141$ (64) $\left[\mathrm{C}_{11} \mathrm{H}_{9}^{+}\right], 98$ (30); HRMS (EI, $80 \mathrm{eV}, 100{ }^{\circ} \mathrm{C}$ ): $\left[\mathrm{M}^{+}\right]: \mathrm{C}_{20} \mathrm{H}_{24} \mathrm{O}_{3}$ calcd.: 312.1725 , found: 312.1735 .

\section{rac-(1R,2R/S,12R,13S)-18-Oxapentacyclo[10.5.2.1 $\left.{ }^{2,6} \cdot 0^{1,13} \cdot 0^{10,20}\right]$ icosa-3,6(20),7,9-tetraen-} 19-one (41)

Analytical data: colorless solid (92\% purity from $\left.{ }^{1} \mathrm{H} \mathrm{NMR}\right) ;{ }^{1} \mathrm{H} \mathrm{NMR}\left(\mathrm{CDCl}_{3}, 500 \mathrm{MHz}\right): \delta$ $=7.16(\mathrm{dd}, J=7.4,7.7 \mathrm{~Hz}, 1 \mathrm{H}, \mathrm{Ar}), 7.05(\mathrm{~d}, J=7.7 \mathrm{~Hz}, 1 \mathrm{H}, \mathrm{Ar}), 6.98(\mathrm{~d}, J=7.4 \mathrm{~Hz}, 1 \mathrm{H}$, Ar), 6.09-6.02 (m, 2 H, 3-H, 4-H), 3.75 (dd, $J=4.8,9.1 \mathrm{~Hz}, 1 \mathrm{H}, 2-\mathrm{H}), 3.42-3.37,3.33-3.27$ $(2 \mathrm{~m}, 2 \mathrm{H}, 5-\mathrm{H}), 3.21(\mathrm{dd}, J=12.1,15.9 \mathrm{~Hz}, 1 \mathrm{H}, 11-\mathrm{H}), 3.12(\mathrm{dd}, J=3.6,15.9 \mathrm{~Hz}, 1 \mathrm{H}, 11-$ H), 2.59 (dd, $J=3.6,12.1 \mathrm{~Hz}, 1 \mathrm{H}, 12-\mathrm{H}), 2.21-2.18,1.62-1.51,1.45-1.38,1.21-1.07,1.00-$ $0.92(5 \mathrm{~m}, 9 \mathrm{H}, 13-\mathrm{H}, 14-\mathrm{H}, 15-\mathrm{H}, 16-\mathrm{H}, 17-\mathrm{H}) ;{ }^{13} \mathrm{C} \mathrm{NMR}\left(\mathrm{CDCl}_{3}, 126 \mathrm{MHz}\right): \delta=181.5$ (s, CO), 137.4, 134.7, 134.0 (3 s, Ar), 127.3, 127.1126 .9 (3 d, Ar), 126.3, 124.9 (2 d, C-3, C-4), 88.0 (s, C-1), 50.1 (d, C-2), 45.3 (d, C-12), 41.9 (d, C-13), 33.9 (t, C-11), 31.1 (t, C-3), 29.5,

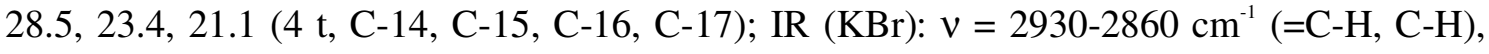
$1760(\mathrm{C}=\mathrm{O}), 1460-1440(\mathrm{C}-\mathrm{H}) ; \mathrm{MS}\left(\mathrm{EI}, 80 \mathrm{eV}, 80{ }^{\circ} \mathrm{C}\right): \mathrm{m} / \mathrm{z}(\%)=280(63)\left[\mathrm{M}^{+}\right], 262(9)\left[\mathrm{M}^{+}\right.$ - $\left.\mathrm{H}_{2} \mathrm{O}\right], 183$ (100), 182 (75), 165 (35) $\left[\mathrm{C}_{13} \mathrm{H}_{9}^{+}\right], 141$ (47) $\left[\mathrm{C}_{11} \mathrm{H}_{9}^{+}\right], 84$ (9); HRMS (80 eV, 80 $\left.{ }^{\circ} \mathrm{C}\right):\left[\mathrm{M}^{+}\right]: \mathrm{C}_{19} \mathrm{H}_{20} \mathrm{O}_{2}$ calcd.: 280.1463, found: 280.1474 .

\section{rac-(1R,2R/S,12R,13S)-16-Ethyleneacetal-18-oxapentacyclo[10.5.2.1 $\left.1^{2,6} \cdot 0^{1,13} \cdot 0^{10,20}\right]$ icosa-}

\section{4,6(20),7,9-tetraen-19-one (43)}

Analytical data: colorless solid; $\mathrm{mp}=190-192{ }^{\circ} \mathrm{C} ;{ }^{1} \mathrm{H}$ NMR $\left(\mathrm{CDCl}_{3}, 500 \mathrm{MHz}\right) \bullet \delta=7.16$ (dd, $J=7.4,7.7 \mathrm{~Hz}, 1 \mathrm{H}, \mathrm{Ar}), 6.98$ (d, $J=7.7 \mathrm{~Hz}, 1 \mathrm{H}, \mathrm{Ar}), 6.97$ (d, $J=7.7 \mathrm{~Hz}, 1 \mathrm{H}, \mathrm{Ar}), 6.40$ (dd, $J=3.3,9.4 \mathrm{~Hz}, 1 \mathrm{H}, 5-\mathrm{H}), 5.95$ (ddd, $J=2.7,6.3,9.4 \mathrm{~Hz}, 1 \mathrm{H}, 4-\mathrm{H}), 3.87-3.74$ (m, $4 \mathrm{H}$, $\left.\mathrm{OCH}_{2} \mathrm{CH}_{2} \mathrm{O}\right), 3.26-3.21(\mathrm{~m}, 2 \mathrm{H}, 2-\mathrm{H}, 11-\mathrm{H}), 3.16(\mathrm{dd}, J=11.5,16.2 \mathrm{~Hz}, 1 \mathrm{H}, 11-\mathrm{H}), 2.84$ (ddd, $J=1.4,6.3,18.1 \mathrm{~Hz}, 1 \mathrm{H}, 3-\mathrm{H}), 2.65-2.59$ (m, $1 \mathrm{H}, 3-\mathrm{H}), 2.56$ (dd, $J=3.6,11.8 \mathrm{~Hz}, 1$ H, 12-H), 2.42-2.37 (m, 1 H, 17-H), 1.85-1.82 (m, $1 \mathrm{H}, 17-\mathrm{H}), 1.74(\mathrm{dd}, J=5.8,12.0 \mathrm{~Hz}, 1 \mathrm{H}$, 13-H), 1.68 (dd, $J=4.4,13.7 \mathrm{~Hz}, 1 \mathrm{H}, 15-\mathrm{H}), 1.61-1.56$ (m, $2 \mathrm{H}, 14-\mathrm{H}, 15-\mathrm{H}), 1.37$ (dd, $J=$ 12.0, $13.2 \mathrm{~Hz}, 1 \mathrm{H}, 14-\mathrm{H}) ;{ }^{13} \mathrm{C} \mathrm{NMR}\left(\mathrm{CDCl}_{3}, 126 \mathrm{MHz}\right): \delta=181.1$ (s, CO), 137.1, 134.1, 132.5 (3 s, Ar), 128.1, 127.7, 125.6 (3 d, Ar), 127.5 (d, C-5), 126.6 (d, C-4), 107.1 (s, C-16), 86.6 (s, C-1), 64.5, 64.3 (2 t, $\left.\mathrm{OCH}_{2} \mathrm{CH}_{2} \mathrm{O}\right), 45.4$ (d, C-2), 45.1 (d, C-12), 40.7 (d, C-13), 36.1 (t, C-14), 33.5 (t, C-11), 29.6 (t, C-15), 27.9 (t, C-17), 23.4 (t, C-3); IR (KBr): v = 3000-2920 $\mathrm{cm}^{-1}$ (=C-H, C-H), $1775(\mathrm{C}=\mathrm{O}), 1450-1435(\mathrm{C}-\mathrm{H}) ; \mathrm{MS}\left(\mathrm{EI}, 80 \mathrm{eV}, 140^{\circ} \mathrm{C}\right): \mathrm{m} / \mathrm{z}(\%)=338(5)$ 
$\left[\mathrm{M}^{+}\right], 320(4),\left[\mathrm{M}^{+}-\mathrm{H}_{2} \mathrm{O}\right], 182(61), 156(100)\left[\mathrm{C}_{8} \mathrm{H}_{12} \mathrm{O}_{3}^{+}\right], 141(76)\left[\mathrm{C}_{11} \mathrm{H}_{9}^{+}\right], 128(14)\left[\mathrm{C}_{10} \mathrm{H}_{8}^{+}\right]$, 99 (26), 43 (2) $\left[\mathrm{OCH}_{2} \mathrm{CH}^{+}\right]$; HRMS (80 eV, $\left.140{ }^{\circ} \mathrm{C}\right):\left[\mathrm{M}^{+}\right]: \mathrm{C}_{21} \mathrm{H}_{22} \mathrm{O}_{4}$ calcd.: 338.1517, found: 338.1536 .

\section{rac-(1R,2R/S,12R,13S)-16-Ethyleneacetal-18-oxapentacyclo[10.5.2.1 $\left.1^{2,6} \cdot 0^{1,13} \cdot 0^{10,20}\right]$ icosa-} 3,6(20),7,9-tetraen-19-one (44)

Analytical data: colorless solid; $\mathrm{mp}=264-266{ }^{\circ} \mathrm{C} ;{ }^{1} \mathrm{H}$ NMR $\left(\mathrm{CDCl}_{3}, 500 \mathrm{MHz}\right): \delta=7.15$ (dd, $J=7.4,7.7 \mathrm{~Hz}, 1 \mathrm{H}, \mathrm{Ar}), 7.05(\mathrm{~d}, J=7.7 \mathrm{~Hz}, 1 \mathrm{H}, \mathrm{Ar}), 6.96(\mathrm{~d}, J=7.4 \mathrm{~Hz}, 1 \mathrm{H}, \mathrm{Ar})$, 6.09-6.01 (m, 2 H, 3-H, 4-H), 3.87-3.83, 3.82-3.73 (2 m, 5 H, 1-H, $\mathrm{OCH}_{2} \mathrm{CH}_{2} \mathrm{O}$ ), 3.45-3.40, 3.32-3.26 (2 m, 2 H, 5-H), 3.22 (dd, $J=12.1,15.9 \mathrm{~Hz}, 1 \mathrm{H}, 11-\mathrm{H}), 3.13$ (dd, $J=3.6,15.9 \mathrm{~Hz}$, $1 \mathrm{H}, 11-\mathrm{H}), 2.63(\mathrm{dd}, J=3.6,12.1 \mathrm{~Hz}, 1 \mathrm{H}, 12-\mathrm{H}), 2.22(\mathrm{ddd}, J=2.2,4.4,14.8 \mathrm{~Hz}, 1 \mathrm{H}, 17-$ H), $1.88(\mathrm{dd}, J=6.0,12.1 \mathrm{~Hz}, 1 \mathrm{H}, 13-\mathrm{H}), 1.72(\mathrm{ddd}, J=4.7,13.2,13.5 \mathrm{~Hz}, 1 \mathrm{H}, 15-\mathrm{H}), 1.62$ (ddd, $J=2.7,6.0,13.2 \mathrm{~Hz}, 1 \mathrm{H}, 14-\mathrm{H}), 1.57-1.49$ (m, $2 \mathrm{H}, 15-\mathrm{H}, 17-\mathrm{H}), 1.42$ (dd, $J=12.4$, $13.2 \mathrm{~Hz}, 1 \mathrm{H}, 14-\mathrm{H}) ;{ }^{13} \mathrm{C} \mathrm{NMR}\left(\mathrm{CDCl}_{3}, 126 \mathrm{MHz}\right): \delta=180.9$ (s, CO), 137.1, 134.3 (3 s, Ar), 127.6, 127.2, 126.9 (3 d, Ar), 126.7, 124.6 (2 d, C-3, C-4), 107.0 (s, C-16), 87.0 (s, C-1), 64.5, $64.3\left(2 \mathrm{t}, \mathrm{OCH}_{2} \mathrm{CH}_{2} \mathrm{O}\right), 49.5$ (d, C-2), 45.2 (d, C-13), 41.5 (d, C-12), 36.3 (t, C-14), 33.9 (t, C11), 29.7 (t, C-15), 29.5 (t, C-5), 29.1 (t, C-17); ); IR (KBr): v = 2975-2860 cm-1 (=C-H, C-H), $1775(\mathrm{C}=\mathrm{O}), 1445-1425(\mathrm{C}-\mathrm{H})$; MS (EI, $\left.80 \mathrm{eV}, 130{ }^{\circ} \mathrm{C}\right): \mathrm{m} / \mathrm{z}(\%)=338(30)\left[\mathrm{M}^{+}\right], 156(100)$ $\left[\mathrm{C}_{8} \mathrm{H}_{12} \mathrm{O}_{3}^{+}\right], 141$ (31) $\left[\mathrm{C}_{11} \mathrm{H}_{9}^{+}\right], 121$ (38), 55 (30), 43 (53) $\left[\mathrm{OCH}_{2} \mathrm{CH}^{+}\right], 28$ (56) $\left[\mathrm{CH}_{2} \mathrm{CH}_{2}^{+}\right]$; HRMS (80 eV, $130{ }^{\circ} \mathrm{C}$ ): $\left[\mathrm{M}^{+}\right]: \mathrm{C}_{21} \mathrm{H}_{22} \mathrm{O}_{4}$ calcd.: 338.1517 , found: 338.1525 . 